\title{
Sustainable operations modeling and data analytics
}

\author{
Angappa Gunasekaran and Nachiappan Subramanian
}

\begin{abstract}
This editorial introduces the unique attributes of this special issue in the era of climate change, modern slavery, and big data. This special issue envisages the depth of penetration of sustainability, from strategy to the operations level, to understand the extent to which sustainability has attracted researchers and practitioners in dealing with various facets of operations management. Overall, it is encouraging to notice the research developments in all facets of operations management except process type, layout type, forecasting, and queuing. Out of three sustainability dimensions, this special issue received substantial contributions on economic and environmental aspects. All the contributions had at least two sustainability components in their decision models as well as newer analytical solutions. At the end, this piece outlines future research challenges and potential research opportunities.
\end{abstract}

Keywords: Sustainable operations, decision model, analytics

\section{Introduction}

Operations management growth is incredible, and it is amazing to see the contributions of researchers from the early nineteenth century to today in applying scientific methods to integrate firms globally to manage production and services, along with the unprecedented growth of technology. Today's business environment expects firms to be flexible and agile to deal with uncertainties in decision-making. Recently, in addition to conventional performance objectives (cost, quality, flexibility, dependability, and reliability), business operations are in a position to incorporate socially and environmentally responsible objectives in decision-making. Fortune 500 firms are green washing to leverage sustainable operations and navigating to turn socially responsible strategic competitive priorities into dominant objectives, in addition to conventional performance objectives. On the research front, the development of sustainable decision models is sporadic, and it is not obvious how far social and environmental objectives are incorporated into the various facets of operations management. 
Some of the potential ways in which different operations facets can incorporate social and environmental objectives are as follows. In product and services design, conventional operational research models are available to check for economic feasibility, design feasibility, and technical feasibility. So far, sustainable objectives are not well taken in the design phase of operations management. Similarly, all facets of operations management mandate sustainability decision models. A few potential research avenues to develop sustainable models within facets of operations management are given below.

Process type : How to include a sustainability dimension in addition to variety and volume while classifying process types.

Layout types : What are the models and methods suitable for incorporating sustainability aspects in layout design?

Logistics : Sustainable decision models for forward logistics, models that incorporate the influence of technology in decision-making.

Capacity : Sustainable capacity decision models that make trade-offs planning between productivity and utilization.

Forecasting : Effect of social and green effects in qualitative and quantitative forecasting.

Production : How to incorporate sustainability factors while developing planning and automated material requirement planning and manufacturing control resource planning. Development of rules to schedule operations based on social and environmental objectives in addition to time and cost.

Operations : Trade-off model to include sustainable practices similar to excellence quality practices to take care of triple bottom line performance.

Zero hazardous

: Models and methods to achieve zero hazardous waste, similar to waste lean methods.

Job design

: Human fair practices decision models in supply chain, benefitcost models for trade-off between triple bottom line performance and productivity.

Queuing : Queuing models considering social and environmental aspects.

Project : Multi-objective sustainable models and methods.

management 
This special issue aims to portray how proactive future operations management should be in the twenty-first century to design their competitive strategy based on social and environmental aspects. In response to our call, researchers contributed their perspective on how to build social and environmental decision models in different facets of operations management.

Most often, recent studies have developed decision models considering environmental and economic aspects without due attention to social aspects. In addition to an economic dimension to achieve ambidexterity in sustainability, coordination between the environmental and social dimensions is essential. Hence, this issue attempts to capture the developments in sustainable decision models in operations management.

Based on our call, we received 44 papers for the special issue. We had a tough time shortlisting the papers, because all the papers submitted were of good quality. Out of 44 papers, $50 \%$ of them - that is, 22 papers - were rejected at the end of first round of review. Finally, the special issue accepted 31.8\% - that is, 16 papers-after two or three rounds of review. We engaged more than 100 reviewers to assess the contributions of the papers submitted to the special issue. We sincerely thank the reviewers for their timely reports and support, which were instrumental for us to successfully complete this special issue.

This editorial is organized as follows: section 2 summarizes the contributions to the special issue as per the operations management facet; section 3 analyses the contribution in terms of sustainable models and data analysis; and finally, section 4 narrates potential future research directions.

\section{Summary of contributions}

We classified the accepted papers as per our call in terms of the following operations facets: process type, layout type, logistics, capacity planning, forecasting, production planning and control, operations excellence, zero hazardous waste, job and work design, queuing, and project management. 


\subsection{Logistics}

Two papers selected under this operations management facet focused on environmental and economic aspects in forward logistics, and the third paper focused on social aspects. The first two papers attempted to develop sustainable decision models considering economic and environmental aspects and relied on standard solvers. However, the third contribution in this facet focused on analytics by proposing a newer neighborhood method. The contributions included food distribution, semiconductor manufacturing, and generic services. Brief introductions to the three articles are as follows.

Soysal et al. illustrated the benefits of horizontal collaboration in reducing total logistics cost and greenhouse gas emissions. The authors developed a decision support model for a food sector involving multiple suppliers and customers considering perishability, energy use of transportation operations, and logistics cost in inventory routing. The authors demonstrated the applicability using a case study from the food sector with two suppliers. The overall outcome of their study indicates that the total reduction of total logistics cost varies from $4-24 \%$ and the emissions vary from $8-33 \%$ depending on supplier size, shelf life, and degree of horizontal collaboration.

Madankumar and Rajendran proposed green vehicle routing problems with pickups and deliveries in semiconductor supply chains. The authors proposed two mixed integer linear programming models, with the first model's objective being to select a set of minimum cost routes and schedules for alternative fuel vehicles that satisfies vehicle capacities and demand requests. In addition, the authors also scaled the model to include the scenario of varying fuel prices at different refueling stations with the objective of operating alternative fuel vehicles with routing and refueling costs. The authors compared the betterment of solution quality and time required to solve the proposed models with conventional models.

Bai et al. have proposed novel efficient neighborhood strategies considering the constraints of a service network design problem (SNDP). Solving a SNDP is a typical challenge, due to the complexity of the constraints and the scale of real-world problems. Hence, the authors derive an efficient transportation network and its 
corresponding schedules using their new neighborhood strategies based on paired route flipping. The authors compared the superiority of the proposed method in terms of solution quality with other high-level meta-heuristics strategies and other generic strategies.

\subsection{Capacity planning}

Two contributions selected under this operations management facet include services with contrasting capacity challenges in the food and maritime industries. The sustainable model in both contributions includes uncertainty and social issues. One paper out of the two selected under this facet proposed a procedure for fair allocation of capacity during competition. Summaries of the two studies are given below.

Hosseinifard and Abbasi discussed the sustainability and resilience issues of a blood supply chain. The authors considered a full-fledged blood chain, including suppliers, blood bank, and hospitals, as two echelons-first on the supply side, with stochastic arrivals of blood from donors, and the second on the demand side, considering the transfusion at hospitals with the role of inventory of perishable products. In particular, the authors show that inventory centralization for perishable products can lead to reduction in waste and shortage. The authors claim their model as novel due to the consideration of uncontrollable replenishment and perishable items in a two-echelon inventory system.

Parthibaraj et al. addressed challenges in the maritime industry by considering flexible freight rates and the coordination of market players with social interest. Hence, the authors developed a sustainable decision model for allocating ship capacity to satisfy shipping demand and generate a feasible route plan. In terms of analytics, the authors proposed multi-agent system modeling and an iterative enumerative combinatorial auction mechanism with Vickrey-Clarke-Groves payments to deploy ships at economically efficient prices. The authors evaluated the models using standard data from the literature.

\subsection{Production planning and control}

Two out of three articles selected under this facet discuss production planning and control issues in manufacturing, and the third one is on aquaculture. All three papers 
developed sustainable decision models including economic and environmental dimensions. In terms of analytics, the first paper by Elaouf et al. uses simulation, the second paper by Fahimnia et al. proposes meta-heuristics algorithms, and the third paper derives policies. Summaries of the three articles are as follows.

Elaouf et al. developed an optimization model to maximize the annual profit of recirculating aquaculture systems, a capital-intensive setup based on construction, equipment, and energy. The authors used a simulation model to replicate the real setup and proposed an optimization procedure using response surface methodology that includes the design of simulation experiments, stepwise regression, and a nonlinear objective function and constraints. The authors' major intention is to develop an effective production planning and operations policy under a given set of conditions that satisfy environmental and economic concerns. Interestingly, the authors open up further research directions by optimizing the biological conditions of aquaculture systems.

Fahimnia et al. developed a model to support production distribution decisions considering economic and emission issues. The authors proposed three meta-heuristic algorithms to solve the non-linear optimization model. The authors illustrated the model using an Australian garment manufacturer. The authors also emphasized the consideration of carbon footprints in production distribution decisions along with cost consideration.

Miao et al. studied the impact of carbon tax policy on manufacturing, which could help multiple stakeholders such as government and manufacturers. In the case of manufacturers, they can decide to what extent they can engage in remanufacturing and decide optimal pricing. On the other hand, policymakers can benefit by using this model to devise a carbon regulation that stimulates producers to engage in remanufacturing as well as to reduce total carbon emissions. The authors' illustration indicates that the increased sales of remanufactured vehicles will reduce the sales of new vehicles, and consequently have a negative impact on the profit of manufacturers. However, the authors argue that in the long term, the regulation can be developed so that it could provide benefits to both manufacturers and society. 


\subsection{Operations excellence}

This facet includes three diverse contributions, one on the service industry and the other two on manufacturing industries. The article on the service industry considers economic and social dimensions of sustainability. On the other hand, the articles on manufacturing industries consider traditional economic and environmental dimensions of sustainability. Interestingly, the paper on the service sector uses an evolutionary game model, the paper on lean manufacturing uses a system dynamics approach, and finally, the third article, on global manufacturing, uses a solution based on the branch and bound technique.

Babu and Mohan proposed a theoretical framework of modeling a supply chain as an evolutionary game, with the focus on social and economic dimensions. The authors addressed gaps in the literature, such as the need of holistic supply chain models including multiple stakeholders to achieve equilibrium over time in terms of economic and social aspects other than exploited environmental issues in sustainable supply chain literature. The authors illustrated the model using a public health insurance supply chain. They suggested including stochastic evolutionary game models in the future.

Gupta et al. used a systems dynamics approach to study the effectiveness of lean thinking with tire manufacturers and to reduce economic and green wastes. The authors' approach could serve as a strategic and operational tool for lean assessment in process industry for practitioners. The approach includes the influence of dynamic changes of resources, such as machine and workforce availability and employees' competence. The authors also find the causation effect of lean and green in a tire manufacturing industry.

Kristianto and Gunasekaran developed a dynamic and stochastic model of global manufacturing as a non-convex, non-linear integer programming model involving topology selection, production capacity planning, transportation routings, and product platform design with product remanufacturing. The authors also proposed a new solution based on the branch and bound method. The unique aspect of the model is the inclusion of pricing, international fiscal regulations, and dynamics of global settings. 


\subsection{Zero hazardous waste}

The paper that discussed close to zero hazardous waste is on capital investment analysis considering carbon emission allowances. The article developed a methodology based on a Bayesian model.

Dhavale and Sarkis developed a methodology that uses a Bayesian model of internal rate of return, which enables decision-makers to consider sustainable factors such as energy efficiency, greenhouse gas reduction, and the value of carbon emission allowances before investing in an asset. The method includes uncertainty and gives an indication to the decision-maker regarding the nature of cash flow as per the nature of the carbon market, such as large and small affecting internal rate of return over a time rather than the unpredictable nature of cash flow. The method would be very helpful for companies in devising sustainability policy initiatives while making internal organizational investment decisions.

\subsection{Job design}

For this facet, we did not find any study directly considering environmental and social issues within an organization, but three studies discussed how to select a partner for a manufacturer. There is no study on the service sector, and all the studies considered the environmental and economic dimensions of sustainability. The authors proposed meta-heuristics algorithms for the first study, hybrid discriminant data envelopment analysis for second study, and mathematical programming for third study.

Banaeian et al. discussed the inclusion of environmental criteria in selecting a supplier. The topic has been investigated in depth by procurement scholars, but not in terms of methodological rigor. Hence, the authors focused on methodological development and incorporated fuzzy environment into three popular methods: TOPSIS, VIKOR, and GRA. Comparison among the methods is made using actual case data from the agri-food industry. The authors found more or less that the three methods reveal a closer result, however, Fuzzy GRA requires less computational complexity. 
Boudaghi and Saen presented a hybrid model using data envelopment analysis and discriminant analysis (DEA-DA) for predicting group membership of suppliers in a sustainable supply chain context. The authors tested their methodology using a car manufacturing company, SAPCO, in Iran. The DEA-DA methodology considers economic, environmental, and social factors. Economic factors considered are experience of suppliers, product quality, delay, price of product, and efficiency of suppliers. Total annual cost of electricity and total annual cost of employee welfare were considered as environmental and social factors, respectively. The authors compared their methodology in terms of prediction precision and proved that the proposed method is far better than existing methodology.

Ma et al. answered some of the challenging issues, such as the influence of carbon taxes on optimal order quantity for a manufacturer, the appropriate range of carbon taxes, and governments' need to implement specific guidelines for manufacturers to select suitable suppliers as per production demand. The authors developed a dynamic programming model by including carbon taxes in deriving optimal order quantity. The procurement-planning model integrated with carbon taxes in a supply chain has been illustrated using the metal industry for selecting appropriate suppliers.

\subsection{Project management}

A study selected in this operations management facet considered all sustainability dimensions in an agriculture supply chain. The authors proposed a two-stage hybrid solution methodology.

Allaoui et al. have developed a multiple objective optimization model and methodology to solve triple bottom line objectives in the agriculture supply chain network. The authors proposed a novel two-stage hybrid solution methodology. In the first phase, they determine the weights using an analytic hierarchy process and ordered weighted averaging aggregation methods. Using the weights, the authors develop multi-objective optimization models and determined pareto-optimal solutions to minimize the total cost of the supply chain network, along with carbon footprint, water footprint, and jobs created. The authors illustrated their model and method using an agro supply chain case study. The authors also mentioned the scalability of their model to sustainable freight transportation. 


\section{Sustainability decision models and data analytics}

Using the studies selected for this special issue, we tried to map the developments of operations management facets on a 2 x 2 matrix, with dimensions of sustainability on the $\mathrm{x}$-axis and the type of data analytics on the y-axis, as shown in Figure 1. It is obvious from the figure that substantial work has been done on the sustainability front focusing on economic and environmental sustainability dimensions, with solution procedures based on mathematical programming or usage of standard optimization packages or simulation-based approaches. Only a few studies attempted to solve economic and environmental dimensions using unconventional approaches. In addition, the operations research community still did not pay much attention to social issues, and there is a tremendous opportunity for scholars to work on the social dimensions of sustainability and on analytical approaches that are either based on mathematical programming or unconventional. Further observations from the contributions show us that the studies are predominantly focused on the manufacturing sector, with a few on service sectors such as public insurance, aquaculture, port logistics, and blood banks. Additionally, we foresee potential research opportunities for modeling and developing potential solutions for the operations management facets of process types, layout types, forecasting, and queuing. 


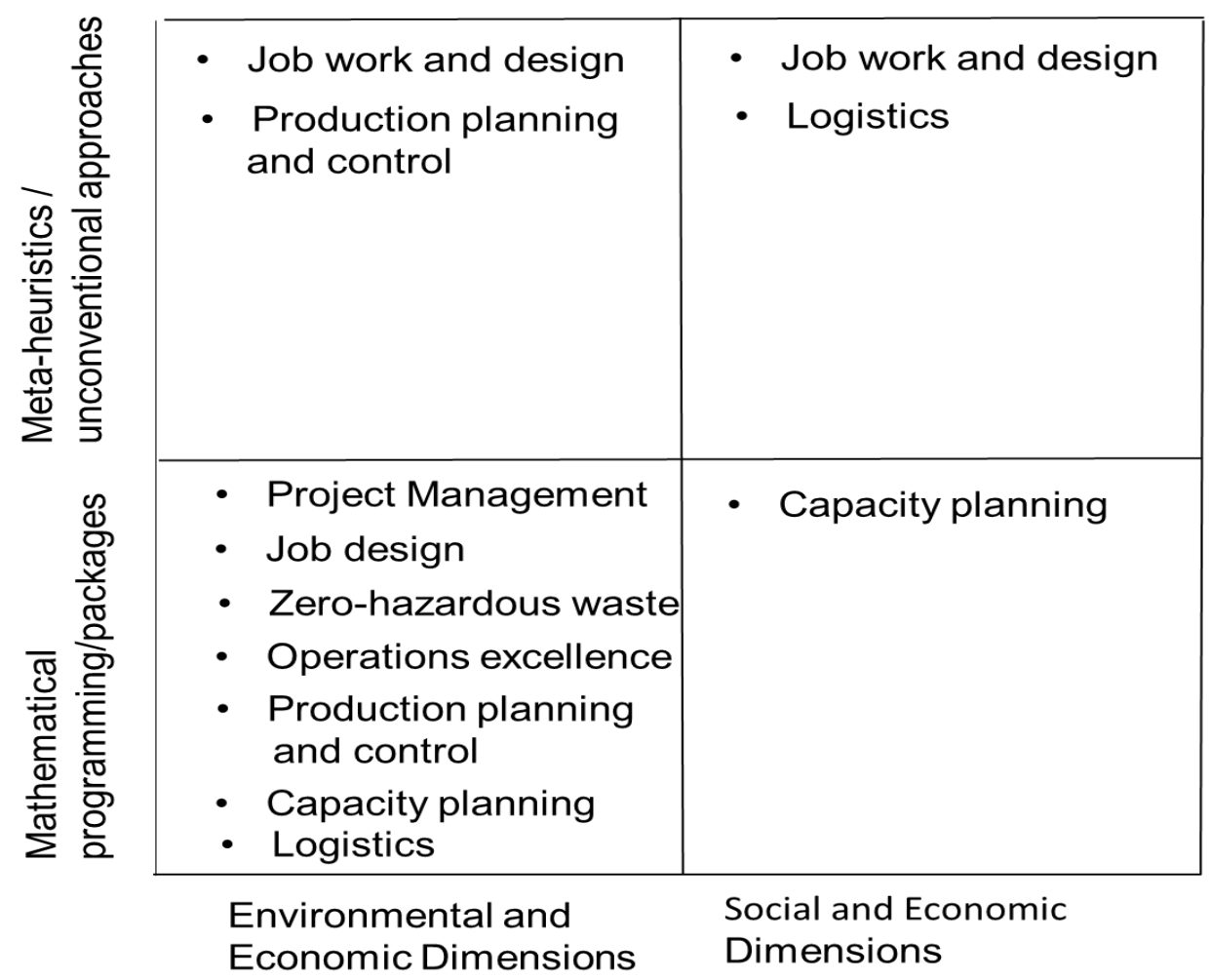

Figure 1: Sustainable modeling and analytics mapping

The framework presented (see Figure 1) illustrates the modeling and analytics mapping for sustainable operations. The modeling and analytics of project management, job work and design, zero hazardous waste, operational excellence, production planning and control, and capacity planning logistics to integrate the environmental and economic dimensions can employ mathematical programming and packages, including optimization solvers, along with data mining and clustering methods. For example, production planning and control models can be developed for a supply chain that will incorporate environmental issues such as energy usage, carbon footprint, reworking, and defective processes and items. Obviously, the environmental and economic dimensions are complimentary to each other and not a trade-off between them. Also, meta-heuristics and unconventional approaches, such as supervised and unsupervised data mining and machine learning techniques combined with evolutionary algorithms — such as ant colony, cuckoo and Tabu search—can be used for incorporating unstructured data (such as big data). Data analytics for job design and production planning and control integrate the various aspects of environmental and economic considerations. 
Social and economic dimensions as an integrated objective has not received due attention in sustainable operations management. Operations management decisions such as capacity planning should include supplier selection and development. Appropriate mathematical programming models can be developed for incorporating suppliers from underprivileged groups, including minorities, women, and the physically disabled, to ensure overall development of the community, not economic interest alone. Job design and logistics should be inclusive of broad-based opportunity for all, while at the same time protecting environmental interests. There are opportunities for big data and analytics applications in job design to deal with modern slavery issues reported in social media by incorporating transparency and inclusion of fair prices. For example, job design should incorporate female and disabled workers so that equal opportunities are available while protecting economic interests.

The application of data analytics becomes more prevalent when we deal with global supply chain operations management. This means that appropriate models and data analytics should be developed and applied to ensure sustainable operations management, taking into account economic, social, and environmental objectives and various constraints. For example, product design plays a major role in controlling the cost of production, but at the same time defining the processes and in turn affecting the environmental, economic, and social objectives.

The initial framework presented here needs empirical validation in order to develop a more accurate framework for sustainable operations management using modeling and data analytics. Some future research directions are listed in the following section.

\section{Concluding remarks and future research directions}

Overall, the special issue attracted several research studies on sustainable operations management, and the predominant research focus is on the following operations management facets: logistics, capacity planning, production planning and control, operations excellence, zero hazardous waste, job and work design, and project management. There is a huge potential for the operations research community to address the following research questions: 
- How can we include sustainability dimensions in addition to variety and volume while classifying process types?

- What are the models and methods suitable to incorporate sustainability aspects in layout design?

- How can we incorporate the modeling and analytics of product design in sustainable operations management?

- The process design plays a major role in sustainable operations; therefore, suitable models need to be developed integrating the big data analytics.

- The global supply network design and operations lead to the handling of big data and analytics. This demands appropriate modeling and data analytic techniques and incorporating the enablers of sustainable operations management.

- What is the effect of social and green effects in qualitative and quantitative forecasting?

- Queuing models considering social and environmental aspects.

- Development of unconventional approaches for social dimensions of sustainability.

- How do we include all three dimensions of sustainability in both the manufacturing and service sectors?

- How do we develop sustainable operations models considering real-time data such as big data?

- How do we develop analytical approaches that are capable of handling structured and unstructured data?

- How do we include uncertainty and risk elements in the manufacturing and service sectors, such as humanitarian logistics?

- Why is there less attention paid to stochastic modeling in the manufacturing and service sectors?

- How do we model transparency in operations and supply chain?

\section{Acknowledgements:}

We are grateful to the two Editors-in-Chief of the Computers and Operation Research Journal for their patience and cooperation throughout the process, especially during 
the change of editorship of the journal. We also express our sincere thanks to the postproduction team for their understanding and kind follow-ups.

\section{Guest Editors:}

Professor Angappa Gunasekaran

Dean and Professor

School of Business and Public

Administration

California State University, Bakersfield

9001 Stockdale Highway, 20BDC/140

Bakersfield, CA 93311-1022

USA

Tel: +1 (661) 654-2184

Email: agunasekaran@csub.edu
Dr. Nachiappan Subramanian

School of Business Management and

Economics

University of Sussex, Falmer

Brighton BN19SL

United Kingdom

Tel: +44 1273872982

N.Subramanian@sussex.ac.uk

Visiting Scholar at Nottingham

University Business School

University of Nottingham Ningbo China

Ningbo 315100, China 